\section{A $\mathfrak{M} \mathfrak{e r t u r e}$}

\author{
MYOPATHY AND A DISTAL FORM. \\ Delivered at the National Hospital for the Paralysed \\ and Epileptic. \\ By Sir W. R. GOWERS, M.D., F.R.S., \\ Physician to the Hospital.
}

Is the last lecture I gave here, I considered the various maladies that depend on defective vitality, on the imperfect vital endurance of the tissues which causes their nutrition to fail, generally early in life, scmetimes later. I suggested that this decay, which results from defect of life, might be called "abiotrophy," and the word has been welcomed as convenient and useful. I illustrated the condition, as some of you may remember, by the wasting of the hair follicles of the skin which causes premature baldness, by the forms of muscular dystrophy which often occur in families, and have thus their essential vital nature strongly emphasized, and also by the many forms of degeneration in the nervous system that are of the same nature, which vary according to the special structure that fails, the time of life at which the vital failure begins, and the cause which sometimes seems to be its incidental excitant.

I have to return to the same subject to-day in one of its special manifestations. We are all opportunists, more or less, and I am able to show you some examples of muscular abiotrophy which are so instructive as to compel me to take them as my subject to-day. You know that these primary affections of the muscles are called "idiopathic muscular atrophy," " myopathy," and also " muscular dystrophy." The last name (due to Erb) means " difficult nutrition," and has a certain aptness, because there is often an overgrowth of the interstitial tissue which suggests the idea of a struggle for existence, such as we sometimes see between weeds and the proper crop which has been sown on the ground. The metaphor is not quite precise, nevertheless we shall see that it is not without a deeper justification. In the form which is termed "pseudo-hypertrophic paralysis," of which I am fortunately able to show you two instructive cases, this interstitial growth attains a special luxuriance. Fat cells are formed in the fibrous tissue this develops, and from this there is an enlargement of some muscles, whence has come the common name of the disease. It was given by l)achenne, who had been specially struck by this feature, the more so because it had chanced to him to meet with some cases in which the enlargement attained a rare extension and degree, being present in most of the muscles. He used to claim Raphael's child-angels as examples of the disease. Some of you may remember the extraordinary development of their muscles as depicted in the celebrated cartoons. In the early days of this hospital there hung, over the fireplace in the consulting-room, an engraving of Raphael's picture, "The Transfiguration"; in it is shown a child with demoniacal possession; it was placed there as an appropriate representation of an epileptic fit, or the most accurate known in art. On a visit Duchenne once paid to the hospital, I remember that he pointed out the figure as one which might well pass for an example of the disease on which he had bestowed the name.

But there may be no increase in size of the muscles, or there may be a decrease. The interstitial tissue may merely compensate for the wasting of the muscular fibres or it may fail to do so. Hence, in the same case, we mpet with muscles that are weak and large, that are weak and normal jn size, and that are weak and small. Interstitial growth, which is luxuriant, or moderate, or slight, is seen in the same case; one or the other may preponderate in different cases. Names are often instructive, but they sometimes obscure. I think that Duchenne's designation hindered the recognition of the fact which has been established by Erb and others that neither the amount of interstitial tissue, nor its character, is an essential element of the disease. The constant element is the change in the muscular fibres themselves; some of them may appear at first unduly large, but many are small and present the features of degeneration; ultimately this be- comes general, irrespective of the increase of the interstitial tissue. It is a true abiotrophy.

As to these differences let me remind you of what I said in the last lecture regarding the growth of interstitial tissue which cccurs in the nervous system when there is primary atrophy of the nerve elements. I pointed out the variations it presents, and that it sometimes attains a luxuriance and energy which make it overstep the strict limits of the degenerated tracts. This feature at one time raised a suspicion that it was the primary element in the process, and a similar suspicion regarding the muscles was excited at first by the abundant development of interstitial tigsue, especially in the pseudo-hypertrophic form. Let me remind you also of another fact 1 pointed out. When weconsider the features of this double process, the atrophy o: the functional structures, and the overgrowth of the interstitial tissue, they suggest that the two elements have a vital relation, common in the necessary connexion of thelife of each, but opposite in tendency. When the nutrition of the muscular or nerve fibres begins to fail, that of the interstitial tissue is increased. It does not grow merely to fill up space; it is not truly a secondary process, it is the expression of an excess of vitality simultaneous with the diminution of that in the proper structures. The two tendencies, plus and minus, seem to be synchronous in these cases of vital failure, and the excess may have results at first more conspicuous than the diminution. The interstitial growth may be at first much more obtrusive than the atrophy of the functional elements. We saw how, in the optic nerve, it. seems to promote the response of the interstitial tiseue to the influence of a toxic agent (tobacco) in the form of interstitial neuritis, the symptoms of which lead the way, but at an earlier age than the toxic influence is alone effective. Moreover, when this influence ceases to act the usual improvement does not occur-the abiotrophy still goes on. The same initial excess of vitality of the connective tissue seems to induce the exuberant development of the interstitial tissue in the muscles, and the production of fat cells within it, in muscular abiotrophy.

The degree of overgrowth varies in degree in different parts and also in different cases, from causes we cannot discern, yet we can understand its features better if we remember the double character of the process. But the excess of the interstitial vitality is only temporary. It often attains a conspicuous degree before the failure of the life of the muscular fibres is considerable, but afterwards, as their failure becomrs great, the vital energy of the interstitial tissue fails also. It is an initial outburst of energy; ultimately it also shares the vital failure. The fat becomes absorbed, and the fibrous tissue gradually contracts, so that the muscles, at first large, lessen but become more firm, and those which are not enlarged shrink ultimately; almost all become small as they become more and more feeble, only some trace of enlargement may linger long where it was at first so great.

We shall see these states in the patients I have to show you. They determine variations in the aspect of cases in which the same muscles suffer in different ways, and still greater variations are due, as we shall see, to the differences in the distribution of the affection. Under the microscope are some sections which are worth your inspection. One is from the deltoid muscle of a case of pseudo-hypertrophic paralysis, and in it you can see the excess of fibrous tissue and the fat cells: between the muscular fibres, and that many of these are narrowed and others are undergoing structural degeneration. Under another is a section of the lumbar enlargement of the spinal cord of the same case; in this you will see that the grey matter and nerve cells present a normal aspect, although the condition of the legs was one of almost c implete loss of power. Under the third microscope I have placed a section of much interest as well as rarity. The part of the section under the microscope looks precisely like a section of muscle in pseudo-hypertrophy. Narrow striated muscular fibres course among tissue composed mainly of fat cells with some strands of connective tissue. But it is a section of a small tumour attached to the spinal cord-a myolipoma. It had caused no symptoms, and was discovered by accident, at least if the phrase can be applied to that which is revealed by a careful search, without definite object. But it is instructive, because it is evidently a fragment of the embryonal 
tissue of muscle which is developed in a wrong place, and in a wrong way. It shows how morbid development results in the condition we find in this disease, and illustrates its truly "abiotrophic" nature.

\section{Pseudo-Hypertrophic Form.}

I'cannot, for lack of time, and I hope I need not, describe the general symptoms of pseudo-hypertrophic paralysis, but I may point out some symptoms that are illustrated by the cases I have here. The first is a bcy of 7 , who is still in the early stage. His case seems alone in his family. I insisted in the last lecture on the fact that all family maladies are prone to occur in isolated form, and how important it is to learn to recognize them by their characters and course, because so often you have not the evidence of their nature afforded by other cases in the same family.

In this child no muscles are sufficiently increased in size to attract much attention, but the calves are distinctly large and firm. The knee-jerks are slight, but are not lost, as they are in the later stage of the disease. $\mathrm{He}$ is just acquiring the characteristic method of getting up from the floor, aiding the extension of the knees by putting his hands on them. This transfers some of the weight of the trunk from the upper end of the femur to its lower end, from the place at which the power exerted by the rectus acts to least advantage, to the place near the fulcrum at which the advantage is greatest. You may ask if this action is due to the weakness of the extensor of the knee, why is it characteristic of this disease, since such these muscles are often weak from other causes The question is a reasonable one. The action is not absolutely characteristic ; it is met with in other maladies, but in such it is very rare. This particular method of aiding the defective movement seems to be acquired only when the weakness comes on slowly during the development of muscular ability. As a matter of fact it is so rare under other conditions that its diagnostic importance is very great.

The boy presents two other sympioms, the significance of which I was, I think, the first to point out. ${ }^{1}$ One is that the infraspinati are enlarged quite as conspicuously as the cal muscles. When the muscle is put in action, as you can see, it appears as a rounded prominence. The other fact is the atrophy of the latissimus dorsi and lower part of the pectoralis major. These sometimes seem absent, and often, as here, are reduced to small dimensions. Their loss is often so great, even at an early stage, as to suggest a congenital defect.

The disease is far advanced in the second boy I show you. His weakness became conspicuous at the age of 7 , and he is now 14. He lost the power of standing four years ago, about a month after an attack of scarlet fever. The acute disease seemed to induce a rapid increase in the symptoms; you should note this, because the symptoms of abiotic disease often first attract attention after some acute disease such as scarlet fever or influenza; and the malady is ascribed to this influence, although it really existed before. It is easy to understand that an affection commencing gradually may have been unnoticed, and that a depressing influence, which really only accelerates, may seem to be the cause. In this boy the calves are not absolutely large, but they are so in comparison with the other muscles, which are extensively wasted, and are weak even out of proportion to the wasting. He has hardly any power of flexing the ankle or extending the knee or hip, but these movements are also prevented by the great contraction which has supervened in the opponents. Permanent shortening occurs very readily in this disease from contraction in the interstitial tissue. When the power of standing has been lost, such shortening quickly fixes the limbs in the position naturally assumed in the recumbent posture. In this child the contraction of the calves is great, and that of the flexors of the knees and hips is such as to prevent extension beyond a right angle. The calf muscles contract earliest and most, doubtless on account of the amount of interstitial tissue in them. The talipes equinus that results often prevents the patient from standing and walking long before the weakness of the other muscles would do so. Once the muscles lose the nutritional influence of use, they become weaker rapidiy, and this weakness, once established, is irremediable. We shall presently see the practical importance of this.

We can still perceive some enlargement of the infraspinati, although less than in the former case; the characteristic atrophy of the latissimus dorsi and lower part of pectoralis is present in an extreme degree. But of all the muscles of his body, that which is enlarged in greatest degree is the lower part of each serratus magnus. You can see how these muscles stand out when he endeavours to push his arms forward. I have seen it before, and indeed it is shown in a figure in my Manual, but it is not common. The infiuences which determine the character of the morbid process in the different muscles are quite unknown to us.

His arms present characteristic weakness, least in the forearms and hands. The muscles of the neck are so feeble that he has some difficulty in holding up his head. The clavicular part of each sterno-mastoid is hardly to be detected, and this is a noteworthy feature, common to many forms of myopathy. ${ }^{2}$ The muscles of the face have here fair power for the most part, but the closure of the eyelids is feeble, and the left orbicularis is much weaker than the right. This is important; the difference between the two sides emphasizes the morbid character of the weakness. I may add that the electrical excitability of the muscles is lessened in both of these patients in proportion to the wasting, equally to each current; this is the characteristic condition in these diseases. In this patient the knee-jerks can no longer be obtained.

\section{Facio-Scapular Form.}

The weakness of the orbicularis is of interest as another of the many links which connect the various forms of muscular dystrophy. It is especially great in a variety in which the face is affected in extreme degree, and likewise are the muscles of the shoulder and upper arm and trunk. As a rule, in this form, wasting accompanies the progressive weakness without conspicuous enlargement. From the distribution it has been called the "facio-scapulo-humeral type," and also, from those who called special attention to it, it has been designated the "type of Landouzy-Dejerine."

I show you now a striking example of this form, and his aspect is strangely similar to that of a patient of whom figures are given in my Manual. I could fancy this was the same patient a few years older. I am able to show him to you by the kindness of my colleague, Dr. Beevor, under whose care the patient is. I must leave it to him to demonstrate the special features, but I would ask you to note the extreme weakness of the orbiculares palpebrarum. It is so great that the patient cannot completely close the eyelids. Common as is the palpebral weakness, in few diseases of this class does it attain the degree seen in this form of myopathy. Yet a characteristic case of myasthenia has lately come under my notice in which the eyelids cannot be brought together by any effort-another link between this myasthenia and myopathy. ${ }^{3}$

Extreme as is the weakness of many of the muscles in this facio-scapular form, great as is the ultimate degree of their atrophy, however long its duration, the malady seems to be purely muscular; the nervous system, even the motor nerves, are unchanged. The fact rests on many observations, on no one more striking than that which has been recently published by Spiller of a case described in the early seventies by Duchenne himself, in which life only terminated at $38 .{ }^{4}$ We might wonder that disuse did not involve structural change in the course of so many years; but we must remember that the inability of the muscular tissue to respond to the motor impulse does not mean that the motor impulses themselves are less than normal. This question, however, would entice me far from my present subject.

\section{Distal MYOPATHY.}

The last case I have to show is one to which I would specially direct your attention because it presents unusual features. Indeed, I have not seen a similar case, nor do I know that one has been recorded, but it is always improbable that any given morbid state has not been seen before, and has not been described somewhere in the vast expanse of medical literature.

This boy is 18 years of age, and is the eldest of three ; the others are healthy. No similar case can be heard of in his family. The symptoms first attracted notice when he was io or 12 years old; then it was noticed that he often caught his toes against the ground in walking. At a later date his hands 
were found to be weak. This feebleness of hands and feet has slowly, steadily increased, until now it is great. He is quite unable to flex the ankles, although he can just extend the toes, and can move each foot slightly in and out by the tibialis anticus and the peronei. He can extend the anklejoint by the calf muscles with some force. The movements of the knee and hip are performed with good power; the kneejerks are present, but the left is less than the right. The thigh muscles are of normal size; the anterior tibial muscles are distinctly smaller on the left side; the calves, on the other hand, are rather large, and are firm. They are sufficiently large to suggest a resemblance to the condition in pseudo-hypertrophic paralysis; they may have been larger in the past, for his mother spontaneously told me that their size had often attracted attention.

A similar state is found in. the upper limbs.

His grasp is extremely feeble. With the right hand he cannot the index to $2 \mathrm{~K}^{\circ}$, instead of 50 or 60 , as he should. Extension is also feeble; he can get the fingers, with the wrist, into line with the forearm, but cannot fully extend them when the wrist is over-extended. This a convenient index of slight deficiency of power in these cases: I had recently occasion to call attention to it in myasthenia, ${ }^{5}$ which is also perhaps a muscular malady. The muscles of the forearms and hands are small, but present no wasting comparable to that which we see in progressive spinal atrophy. Above the forearm the muscles have fair power and present no wasting; only a triffing atrophy of the middle part of the trapezii can be observed. In the affected muscles electrical excitability is lowered in proportion to their feebleness, and equally to faradism and voltaism. To this there is one curious exception; although the muscles in front of the lower leg are notably smaller on the left side, the peronei and tibialis anticus react more readily than on the right side.

But this is not all. His neck muscles are normal, with one noteworthy exception-the sterno mastoids. Of these only a trace remains; only a small fasciculus of the sternal part can be put in action by the will or electricity. The clavicular part seems quite absent. The condition is of such a character, and is so similar on the two sides, as to suggest rather a congenital defect than an actual atrophy. It is a feature conmecting the condition with other myopathies. We have just seen deficiency of this part in paeudo-hypertrophic paralysis. ${ }^{8}$ The platysma here also seems absent.

The condition of his face is noteworthy. The movement of the lips seems unimpaired. His tongue, however, presents a curious aspect of wasting on each side, yet it moves properly, and there is no impairment of the palate. His smile, especially on the right side, presents too little movement outside the corner of the mouth in proportion to the elevation of the apper lip. But the most important weakness is in the upper part of the face. He cannot raise the eyebrows at all; the frontales are powerless. Closure of the eyes by the orbiculares is also weaker than normal ; their contraction is easily overeome, and more easily on the left side than on the right, conclusive evidence that it is morbid. This weakness of the orbiculares is met with, as I have said, in many forms of myopathy : it is thus of considerable significance, and so are the weakness of the frontales, and the atrophy of the sternomastoids. All these features stamp this case as a primary myopathy, and yet it differs from all recognized forms in the purely distal distribution of the affection in the limbs, and the normal state of the muscles near the trunk. In other forms the preponderant weakness and wasting are in the muscles moving the proximal parts, of the shoulder girdle and the hip, of the elbow and the knee. In the upper limb this is very conspicuous. In the leg the affection of the calf muscles is usually attended with weakness of the flexors of the ankle, but even in the early stage the loss of power in the thigh muscles and those of the hip is greater, and as the disease advances the preponderance of their atrophy becomes more and more manifest. Yet in this lad these muscles have good power, while any flexion of the ankle-joint is impossible, not from contraction of the calves but simple failure of the flexors. The feet ean be moved inwards and outwards, and the movement outwards by the peronei is a distinction from the "peroneal form" of muscular atrophy, which, moreover, is believed not to be a true myopathy. Peculiar as is the dis- tribution of the weakness the symptoms in the face and neck associate it with other forms and compel us to regard it as of the same nature, an example of what we may term "distal myopathy."

It is indeed remarkable how many symptoms, small we may regard them, link together the various forms of myopathy, and how important therefore is their recognition. Not less remarkable is the way in which the general type is maintained in families in which several cases occur, and hardly less important are the variations from the type which individual cases frequently present. These variations leave the general character of the malady clear, and yet they connect one type with another. I wish I could show you another case, which affords a pertinent illustration of this connexion-so pertinent indeed that I must tell you the chief features. The distal form, with weakness of the hand only, which I have just shown you, presents a marked contrast to the facio-scapular form in which the shoulder muscles suffer and upper arm muscles suffer chiefly. But in this case the affection of the face is slight, and instead of it the hand muscles have suffered, in addition to those of the scapula and upper arm. She is a girl of 23, the seventh of a family of eleven, all of the same sex, a fact to note. Three of them-the first, second, and fifth -suffer from a similar disease; three others died in early life; four are healthy. ${ }^{*}$ Their parents were not related, and no like disease can be heard of in the family. Yet the affection of four sisters shows how such a vital tendency may be congenital in a family, though not inherited. Some difficulty in walking, in raising the toes, was first noted at 16 , and weakness in the arms at 18 . The affection of the shoulder girdle and upper arm here is precisely that of an advanced case of the Landouzy form. Little trace remains, in substance or in power, of the trapezii, serrati, latissimi, or pectorales. The upper arm muscles are thin and feeble, especially on the right side; the deltoids retain some substance.

Equally great, however, is the affection of the distal muscles. We find no trace of the supinator longus. The flexors of the wrist and fingers retain some power; the extensors hardly any. Even the interossei are shrunken and very weak ; if the first phalanges are passively extended, the others can only just be straightened by an effort. The metacarpal bone of the thumb can be well bent back, but no extension of its phalanges is possible. Everywhere, I may say, electric excitability is diminished with power, and alike to each current, as is the rule in myopathy. Thus in the upper limbs the condition is that of the scapulo-humeral form, but there is an unusual degree of affection of the distal part of the limb.

In the legs the affection is almost limited to the muscles in front of the lower leg. The movements of the hip and thigh are good and strong, and the knee-jerks are active. The foot can be pushed down by the calf muscles, which are of moderate size and not hard. But there is no power of flexing the ankle. The right foot can be moved in and out feebly, but the left can only be inclined inwards. Thus the affection of the legs is purely distal, as in the boy I have shown you; the proximal muscles are normal. In the arms the affection is both proximal and distal. Yet these two cases are linked together, and connected with other forms by common features.

Pathology and Treatment.

I have spoken of all these cases as myopathic maladies, as muscular abiotrophy, the result of a defective vital endurance,

* Mr. H. Burland of Finedon, has kindly furnished me with some particulars of the family of the case last mentioned. Not only does it consist of eleven girls and one boy, but the mother was one of a family of twelve girls; all the girls, except herself, died in early life. Of the patient's sisters, in one, now 30 years of age, difficulty in walking was noted first at 15, after messles, that of the arms only at 27. She can now walk only with much difficulty. Movements of the hips and knees are weak, so are flexion and eversion of the ankle, but inversion is strcng. The knee-jerks are absent. The flexors and extensors of the hands and fingers are weak but not much wasted. The upper arm muscles are small and feeble, but not the deltoid. The trapezii, serrati, and latissimi are all wasted. The clavicular part of the sterno-mastoid is good. The orbiculares palpebrarum are feeble, the frontales strong. In the other sister, now 25 , weakness in walking was noticed at 13, after sore throat. In her the frontales have no power and the orbiculares are weak, and both parts of the sterno-mastoids are small. The muscles of the shoulder, elbow, and hand are all good, but there is some impairment of free movements. In the legs there is very little weakness, but the knee-jerks are absent doubtful. 
inherent in the embryonal tissue from which the muscular structures of the body arise-a defect variable in distribution, in character, and in tendency, but essentially the same in all. In a few cases trifling changes have been found in the nervous system ; in most it has been absolutely normal, both nerves and central structures, even by the latest methods of research, and in cases of most prolonged duration and profound degree. This fact seems effectually to dispose of the hypothesis that the muscular change is in any way the result of the trifling changes that have been found in a few cases, or the more considerable atrophy of the spinal nerve cells found in one case. When we remember the fact, which I pointed out in the last lecture, that abiotrophy is met with in the nervous system, in various structures, and of various course, it is strange that it is not more of ten met with as an associated state. It is not surprising, moreover, that slight secondary changes should be met with in the spinal cord when there has been prolonged secondary spinal curvature, with all its effects on the circulation. Such trifling changes, met with in a few cases, irregular in seat and distribution, can have no real significance. Yet they have been thought to suggest that some day it may be discovered that the muscular condition depends, after all, on a morbid state of the spinal cord. This idea is a relic of the old fancy, for it was nothing more, that there are in the spinal cord special trophic centres for the muscles apart from the motor cells of the anterior cornua. It is a doctrine that should have died of inanition long ago ; purely hypothetical, every definite fact is opposed to it.

The vitality of the muscles presents a strange duplicity. Perhaps it is not so mysterious as it seems at first view; the same double relation may be seen, I think, elsewhere, although less clearly. The nutrition of the muscle depends on that of the nerves through which its function is called forth. If the nerves slowly degenerate, so does the muscle ; if rapidly, from descending irritation, the muscles undergo speedy complete degeneration. Yet the muscle has a life which we may call organic, belonging to it as a structural entity, in consequence of which it may undergo morbid changes, apart from the nervous system, and may fail to live on, though the nerves preserve an unimpaired vitality. This failure is what I have called muscular abiotrophy-failure of nutrition from defective vitality-and for brevity we call it myopathy.

The treatment of this condition is a narrow subject, yet not unimportant. Its importance is positive and negative; to know what we cannot do and what we can. Life itself is beyond the influence of medicinal agents. Some degenerative changes seem to be within control at least in some degree, but it is otherwise with those vital tendencies which seem inherent. We can discern no clear evidence that they can be thus influenced; we can perceive no positive effect from the administration of medicine of any kind, and no deterioration when such agents are omitted. Yet even vital energy is not altogether beyond indirect influence. There is one agency, but only one, which has a sure effect on the vital nutrition of the muscles, and that is their voluntary use, their stimulation through the nerves with which their nutritional integrity is so mysteriously bound up. That is the lesson taught by all true muscular growth, by the disciples of Sandow, as by every athlete. The application of the lesson to these diseases is all-important; it is seen alike whether it is enforced or disregarded.

Muscular exercise, adapted in degree to the muscular state is the one agent which distinctly stems the ebbing tide of life, and hinders the failure of muscular strength and muscular nutrition. Neither electricity nor massage can exert more than a small fraction of the influence of voluntary use. Without it no definite effect can be observed from their em. ployment; with it they seem sometimes to do a little good, and upward massage promotes the circulation in the muscles and the renewal of the blood plasma, from which their nutritional elements are derived. But that is all we can say.

When contractures or any accidental causes arrest the voluntary use of the muscles for even a few months, the weakness and the wasting increase rapidly, and speedily reach $a$ degree from which there is no recovery. Contracture occurs readily in the muscles in which there is much increase of consective tissue, especially in the calf muscles, but the tendency to it varies. In some cases it prevents standing and walking long before this would be lost from the weakness of the muscles of the upper leg. Then the divisinn of the Achilles tendons is imperative. I have mentioned ${ }^{7}$ one case in which this contraction occurred so $\mathrm{far} / \mathrm{y}$, in sach degree, that at five years of age the ability to stand would soon have been lost from this alone; tenotomy enabled the patient to walk until 12 , and then again became necessary. It preserved the ability for years; at 20 the patient could still walk three miles.

When the contraction occurs late, and the weakness of the muscles of the knee and hip is great, the question may arise whether the rest the operation entails will not augment the weakness, gn that the effect of tenotomy is useless. But the rest need be but brief, and its effect may be lessened by movements while lying. If the contraction is not extreme it may be diminished without tenotomy by an apparatus $I$ devised some years ago, ${ }^{8}$ in which elastic traction on leather around the foot is made from leather around the leg, the two being united by a narrow piece behind the heel; this bends, but has sufficient vertical resistance to prevent pressure on the skin, which, if continuous, so soon becomes intolerable. Worn during rest and indoors, the effect of the very gentle constant elastic traction is most remarkable.

At the knee and hip the contraction is of the flexors, and is the speedy consequence of posture which involves flexion of these joints as the patient sits or lies. It usually attends a degree of weakness which precludes any prospect of good from operation. It emphasizes only too strongly the importance of maintaining as long as possible the upright posture. When the patient can only sit, not only do these contractions of the hip and knee occur, but the weakness of the muscles of the trunk induces inevitably an inclination to one side, which leads to curvature of the spine, with all its consequences on the movements of the chest, and the disposition to chronic changes in the lungs by which most sufferers find the end of life.

Elsewhere, in the arms especially, the essential element in treatment is the same persevering efforts to maintain the exercise of the muscles. For this gentle gymnastic exercises are extremely useful, especially the various' apparatus for developing the muscles of the arms. But it is difficult to secure the necessary perseverance without the incentive which games provide. Fencing, indeed, is a most effective exercise when the fallure of the arms is slight, if the exertion is moderate and the object kept in view. Cycling gives some exercise to the arms as well as to the legs, and a tricycle is often practicable and most useful when a bicycle cannot be ridden. Lawn tennis involves too severe effort to be commonly available, but its degraded indoor form of ping-pong necessitates the varied but gentle use of many muscles, which makes it far more than a mere game to sufferers from this disease, and it is additionally useful if played in the open air. When the movements of the hand begin to be impaired, the action of playing the piano is very useful, and the art should be acquired in anticipation of its need, that the pleasure should be an incentive to exercise, as it can scarcely be in the stage of laboured acquisition.

It is the case with all measures which merely hinder a progressive malady - their influence, however great, is unobtrusive. It is negative, and not positive. How real and important it has been is only perceived when it ceases. The speed with which the malady then advances reveals the effect that had been unperceived. The sufferer and his friends are inapt to realize these negative results, and apt to think that no good is done because no improvement is perceived. We may do something to save them from this error, and we must be careful not to fall into it ourselves. We should also do our best to try to save them from efforts to obtain good from the many straws held out for hope to grasp at, which are magnified by promise into rods of rescue. Those who promise with assurance that which cannot be, always find too ready credence, as is often only found too late.

\section{NOTES AND REFERENCES.}

1 Pseudo-hypertrophic Muscular Paralysis. London: Churchill. 1879 2 I noted this feature as long ago as 1879.3 BRITISH MEDICAL JOURNAL, May 24th, 1902. 4 Proc. Pepper Jab., Philadelphia, 1500. 5 Loc. cit. ${ }^{6}$ trophic Paralysis, Case 35 , and p. 55. ${ }_{8}$ Clin. Lect, ed. 1895, p. 394. 